\title{
Preventable deaths in patients with traumatic brain injury
}

\section{Seong Chun Kim', Kyoung Jun Song ${ }^{2}$, Sang Do Shin ${ }^{2}$, Seung Chul Lee ${ }^{3}$, Ju Ok Park ${ }^{2}$, James F. Holmes ${ }^{4}$}

'Department of Emergency Medicine, Gyeongsang National University Hospital, Jinju, Korea ${ }^{2}$ Department of Emergency Medicine, Seoul National University Hospital, Seoul National University College of Medicine, Seoul, Korea

${ }^{3}$ Department of Emergency Medicine, Dongkuk University College of Medicine, Ilsan, Korea ${ }^{4}$ Department of Emergency Medicine, UC Davis Medical Center (JFH), Davis, CA, USA

Objective The objective of this study is to evaluate the rate of and etiology for preventable deaths in patients with traumatic brain injuries (TBIs).

Methods We conducted a retrospective, multicenter review of patients with TBIs who died within 7 days of their traumatic event from June 2008 to May 2009. Three board certified emergency physicians independently reviewed every case using a structured survey format. Cases were considered preventable deaths only if all physicians independently agreed the death was preventable. Management errors contributing to the preventable death were determined.

Results Forty-one patients who died from TBI were eligible. Preventable deaths were identified in nine (22\%; 95\% confidence interval [Cl], 11 to 28) cases. Fifty-six management errors were identified including $36(64 \% ; 95 \% \mathrm{Cl}, 50$ to 77$)$ in the emergency department and $13(23 \%$; $95 \% \mathrm{Cl}, 13$ to 36$)$ in the prehospital phase. Thirty (54\%; 95\% Cl, 40 to 67$)$ management errors were process-related, and 26 (46\%; 95\% Cl, 33 to 60) were structure-related.

Conclusion An important and measurable rate of preventable mortality occurs in the initial care of TBI patients. Errors were common and most occurred in the emergency department. In addition, errors were common in the prehospital phase but did not always lead to mortality. When analyzed by type of problem, both process-related and structure-related errors occurred in similar proportions.

Keywords Death; Brain injury; Errors; Process; Structure

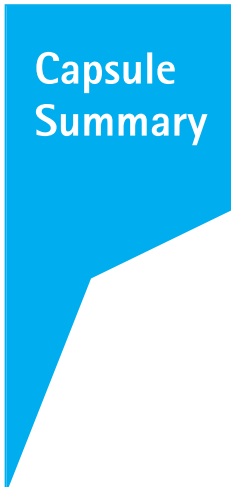

What is already known

Preventable death rate is currently a method to determine the performance of emergency medical system for trauma. Some reports from Korea showed the preventable death rate ranges from $30 \%$ to $40 \%$.

What is new in the current study

Previous studies on preventable traumatic death focused on all severely injured trauma patients regardless of the presence of traumatic brain injury. We identified the preventable death rate and etiology in patients with traumatic brain injury.
Received: 17 July 2014

Revised: 25 December 2014

Accepted: 8 January 2015

Correspondence to: Kyoung Jun Song Department of Emergency Medicine, Seoul National University Hospital, Seoul National University College of Medicine, 101 Daehak-ro, Jongno-gu, Seoul 110-744, Korea

E-mail: skciva@gmail.com

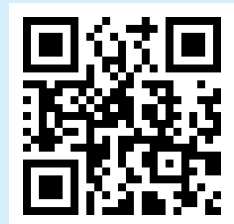

How to cite this article:

Kim SC, Song KJ, Shin SD, Lee SC, Park JO, Holmes JF. Preventable deaths in patients with traumatic brain injury. Clin Exp Emerg Med 2015;2(1):51-58.

This is an Open Access article distributed under the terms of the Creative Commons Attribution Non-Commercial License (http:// creativecommons.org/licenses/by-nc/3.0/). 


\section{INTRODUCTION}

In 2009, 32,661 people died from injuries in Korea, making it the third leading cause of death after cancer and cardiovascular disease. ${ }^{1}$ Furthermore trauma is the leading cause of death and disability in those one to 40 years of age and is the leading cause of years of productive life lost and economic lost. ${ }^{1}$ Preventable deaths include those deaths which would not have occurred had the patient received appropriate care in a timely fashion. The preventable trauma death rate is currently a method to determine the performance of a country's or region's emergency medical system. ${ }^{2}$

A prior Korean study of 131 trauma deaths from two tertiary and four secondary hospitals suggested the preventable death rate may be as high as $40.5 \% .^{3}$ Furthermore, in this study, the preventable death rate was 22\% for those with traumatic brain injury and 63\% in those without brain injury. Data from three regional and six local Korean hospitals suggested a preventable death rate of $40 \%$ but may be as high as 56\% in those with traumatic brain injury. ${ }^{4}$

Many cases of traumatic brain injury require emergent neurosurgery and without appropriate, timely treatment, secondary brain injury may rapidly occur. ${ }^{5,6}$ Previous studies on preventable trauma death focused on all severely injured trauma patients regardless of the presence of traumatic brain injury. The objective of this study is to identify the preventable death rate and reasons in patients with traumatic brain injury.

\section{METHODS}

We conducted a retrospective study of adult patients with traumatic brain injury treated at five hospitals in the Korean Traumatic Brain Injury Surveillance Network. Two in five hospitals were regional emergency medical centers and three of them were local emergency medical centers. The study was approved by the institutional review boards at all sites.

\section{Study population}

Patients older than 15 years of age who were treated for traumatic brain injury and died within 1 week from their initial presentation between June 1, 2008 and May 31, 2009 were included. The patients had traumatic brain injury but, could have other injury also. The definition of traumatic brain injury followed the International Classification of Diseases 10th edition (ICD-10) and included patients with any of the following codes: S01.0-S04.0, S06.0-S07.9, S09.7-S09.9, T01.0, T02.0, T04.0, T06.0, T90.1-T90.9. This corresponds to patients with brain hematomas (including intracerebral, epidural, and subdural hematomas), contusions, hemorrhage, or cerebral edema.
Patients were excluded if they received cardiopulmonary resuscitation on arrival to the hospital or if cranial computed tomography scanning was not performed. In addition, we excluded patients with incomplete medical records where we were unable to determine either the time of death or if the death was preventable.

\section{Study protocol}

A preventable death was defined as a death that is preventable if appropriate treatment was provided in a timely fashion. To determine if the patient with traumatic brain injury experienced a preventable death, the following three criteria were required. ${ }^{2}$ First, the traumatic brain injury must be of such a degree that the patient is viable (i.e., the traumatic brain injury cannot be severe enough to cause death). Second, the treatment provided to the patient must be considered insufficient compared to standard treatment of traumatic brain injury as provided in the Advanced Trauma Life Support (ATLS) course guidelines and the Brain Trauma Foundation (BTF) guidelines. ${ }^{7.8}$ Finally, the insufficient treatment must contribute to the death of the patient either directly or indirectly.

Traumatic deaths were divided into three categories. ${ }^{9}$ First, nonpreventable deaths include those injuries so severe they are not amenable to current medical care. These include patients with injuries categorized as Abbreviated Injury Scale (AIS) score of 6. ${ }^{10}$ Second, potentially preventable deaths are those with very severe injuries, but are survivable with the current level of medical treatment. An example of a potentially preventable death would include a patient with a massive subdural hematoma who arrives at the hospital in time for emergent surgical decompression however, the patient died as they were transferred to a hospital without emergent neurosurgical capabilities. Thus, the injury was potentially preventable if the patient had initially been transported to an appropriate hospital. Finally, definitely preventable deaths are those injuries that are treatable based on the type of brain injury and capabilities of the initial treating hospital. An example of a definitely preventable death is the patient who arrives at a hospital with the neurosurgical capabilities to treat an epidural hematoma but the patient dies of brain herniation prior to identification of the epidural hematoma (delayed diagnosis).

We considered preventability in context to the following: injury severity, condition of the patient, and adequacy of treatment. Injury severity was based on the AIS score. Patient condition was based on the Revised Trauma Score. The presence of underlying disease and complications was determined from review of the medical history. Adequacy of medical treatment was based on treatments recommended in the ATLS course and the BTF guidelines. Through this process, cases were classified into one of three categories; defi- 
nitely preventable, potentially preventable, or non-preventable.

Treatment deficiencies were divided into the following six 'location' categories: prehospital phase, transfer phase between hospitals, emergency department phase, operating room phase, intensive care unit phase, and ward phase. Treatment deficiencies were further divided into structure-related problems and processrelated problems. Structure-related problems included those secondary to an absence of appropriate facility, personnel, or equipment for timely diagnosis and treatment. Process-related problems included those due to inadequate treatment, diagnosis, or procedure.

We used an expert panel (professional study panel) composed of three specialists in emergency medicine to determine the outcome of interest. The medical records (including all radiographs and treatment) were reviewed independently by each member of the team.

The team underwent two formal training sessions prior to performing the record review. The research team visited each hospital, reviewed the medical records and radiographic images of all eligible patients and independently determined the outcomes.

We further categorized all potentially preventable and definitely preventable deaths into those that occurred at any point in treatment (i.e., both prehospital and hospital phase) and those occurring only during hospital treatment. Preventable/definitely preventable deaths during hospital treatment were determined based on the patient's condition upon arrival at the hospital. After the outcomes were determined by each member of the research team, agreement between investigators was determined.

\section{Data analysis}

We calculated the preventable death rate among all traumatic brain injury patients including those occurring at the hospital lev-

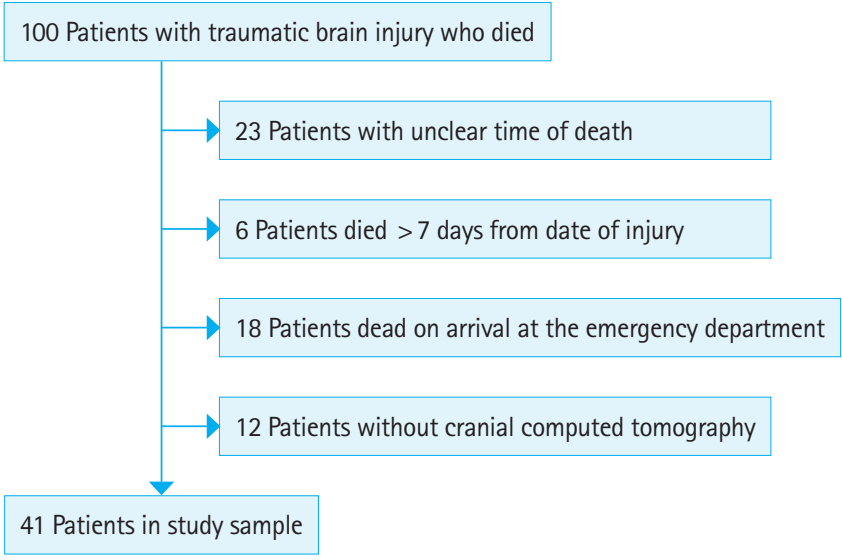

Fig. 1. Among 100 patients with traumatic brain injury, 59 patients were excluded and 41 patients were enrolled finally. el. We also calculated the preventable death rate by patient characteristics including expected probability of survival based on ICD10 injury severity score (ICISS) including patient's gender, age, and mechanism of injury.

A preventable death was only considered present if all investigators independently agreed. Results are described with simple descriptive statistics. Categorical data are compared with Fisher's exact test due to small cell size. The degree of agreement between investigators was measured with weighted Kappa values and 95\% confidence intervals (Cls) were calculated. We additionally collapsed the definitely preventable and potentially preventable categories into a single category (preventable deaths). Alpha Xi Bach (Cronbach's alpha) reliability coefficients were calculated for cases analyzed to three categories (definitely preventable, potentially preventable, or non-preventable) and two categories (preventable deaths or non-preventable) to determine internal consistency.

\section{RESULTS}

\section{General characteristics of the population}

A total of 27,409 trauma patients were evaluated at the study sites from June 1, 2008 to May 31, 2009 and 7,131 patients had head trauma. One hundred (1.4\%) of the 7,131 patients died. Fifty-nine patients were excluded (Fig. 1). Therefore, the study population consists of 41 patients who died from traumatic brain injury during the time period. Characteristics of the study population are presented in Table 1. The median age was 58 years (interquartile range, 43 to 73 years). The median probability of survival was $64 \%$ (interquartile range, $57 \%$ to $79 \%$ ).

Table 1. General characteristics of the 41 patients

\begin{tabular}{lc}
\hline Charateristic & Value \\
\hline Male sex & $25(61.0)$ \\
Age $<55$ yr & $18(43.9)$ \\
Mechanism of injury & \\
Motor vehicle collision & $24(58.5)$ \\
Fall & $14(34.2)$ \\
Assault & $1(2.4)$ \\
Unknown & $2(4.9)$ \\
Cause of death & \\
Brain injury & $27(65.9)$ \\
Hemorrhage & $6(14.6)$ \\
Multiple injury & $7(17.1)$ \\
Others & $1(2.4)$ \\
Probability of survival by ICISS (\%) & \\
$<50$ & $7(17.1)$ \\
$50-75$ & $21(51.2)$ \\
$>75$ & $13(31.7)$ \\
\hline
\end{tabular}

Values are presented as no. of patients (\%).

ICISS, International Classification of Diseases 10th edition injury severity score. 
Table 2. Potentially and definitely preventable deaths

\begin{tabular}{|c|c|c|c|c|c|}
\hline & \multirow{2}{*}{$\begin{array}{l}\text { No. of } \\
\text { patients }\end{array}$} & \multicolumn{2}{|c|}{ All causes } & \multicolumn{2}{|c|}{ Hospital causes } \\
\hline & & PP & DP & PP & DP \\
\hline Total & 41 & $4(9.8)$ & $5(12.2)$ & $3(7.3)$ & $4(9.8)$ \\
\hline \multicolumn{6}{|l|}{ Cause of death } \\
\hline Head trauma & 27 & $0(0.0)$ & $5(18.5)$ & $1(3.7)$ & $4(14.8)$ \\
\hline Non-head trauma & 14 & $4(28.6)$ & $0(0.0)$ & $2(14.3)$ & $0(0.0)$ \\
\hline \multicolumn{6}{|c|}{ Probability of survival by ICISS (\%) } \\
\hline$<50$ & 7 & $0(0.0)$ & $1(14.3)$ & $0(0.0)$ & $0(0.0)$ \\
\hline $50-75$ & 21 & $2(9.5)$ & $3(14.3)$ & $2(9.5)$ & $3(14.3)$ \\
\hline$>75$ & 13 & $2(15.4)$ & $1(7.7)$ & $1(7.7)$ & $1(7.7)$ \\
\hline
\end{tabular}

Values are presented as no. of patients or $n(\%)$.

$\mathrm{PP}$, potentially preventable; $\mathrm{DP}$, definitely preventable; ICISS, International Classification of Diseases 10th edition injury severity score.

\section{Preventable death rate}

Nine (22\%; 95\% Cl, 11 to 28 ) of 41 cases were determined to be potentially or definitely preventable. This included five (12\%; 95\% $\mathrm{Cl}, 4$ to 26$)$ cases definitely preventable and four $(10 \% ; 95 \% \mathrm{Cl}$, $3 \%$ to $23 \%$ ) cases potentially preventable. Seven (17\%; $95 \% \mathrm{Cl}$, 9 to 25 ) cases of preventable deaths occurred secondary to errors in the hospital, including four (10\%; $95 \% \mathrm{Cl}, 3 \%$ to $23 \%$ ) cases definitely preventable and three $(7 \%, 95 \% \mathrm{Cl}, 2 \%$ to $20 \%)$ cases potentially preventable (Table 2 ). The remaining two cases of potentially preventable deaths occurred prior to hospital arrival. The median age of patients with potentially/definitely preventable deaths was 70 years (interquartile range, 58 to 80 years).

As we focused on variables that influence preventable trauma death, preventable death rate was lower in the case of death caused by head trauma compared to non-head trauma $(P=0.692)$. At the hospital level, it was higher in the case of death caused by head trauma $(P=1.00)$. However both were not statistically significant.

When the 'potentially preventable' and 'definitely preventable' categories were collapsed into a single 'preventable' category, the preventable death rate was lowest (14.3\% at the overall phase and $0 \%$ at the hospital phase) when probability of survival by ICISS was less than 50\%. Interestingly, preventable death rate was highest (23.8\% both at the overall and hospital phase) when probability of survival by ICISS was between $50 \%$ and $75 \%$.

\section{Errors related to the treatment process}

Fifty-six treatment errors were identified in the 41 patients (1.4 errors/patient). Locations of errors were as follows: emergency department 36 (64\%), pre-hospital phase 13 (23\%), interhospital transfer 4 (7\%), and intensive care unit 3 (5\%). Types of problems include structure-related 26 (46\%) and process-related 30 (54\%). Process-related errors determined to be directly related to the death occurred in 26 (58\%) cases. A list of the errors identified is presented in Table 3 (Appendix 1 for the details of actual errors
Table 3. Management errors by location and type

\begin{tabular}{lccc}
\hline & Total (A) & Related to death (B) & $\mathrm{B} / \mathrm{A} \times 100(\%)$ \\
\hline Total & $56(100.0)$ & $45(100.0)$ & 80.4 \\
Location & & & \\
$\quad$ Prehospital phase & $13(23.2)$ & $7(15.6)$ & 53.8 \\
Interhospital transfer & $4(7.1)$ & $4(8.9)$ & 100.0 \\
Emergency department & $36(64.3)$ & $31(68.9)$ & 86.1 \\
$\quad$ Operating room & $0(0.0)$ & $0(0.0)$ & - \\
Intensive care unit & $3(5.4)$ & $3(6.7)$ & 100.0 \\
$\quad$ Ward & $0(0.0)$ & $0(0.0)$ & - \\
Type & & & \\
Structure-related & $26(46.4)$ & $19(42.2)$ & 73.1 \\
$\quad$ Process-related & $30(53.6)$ & $26(57.8)$ & 86.7 \\
\hline
\end{tabular}

Values are presented as $n(\%)$ unless otherwise indicated.

and Appendix $2^{3,4}$ for the problem codes to classify the problems).

\section{Agreement among investigators}

Kappa values demonstrated moderate agreement between reviewers (kappa $=0.46$ for overall preventable death and 0.43 for hospital preventable death). Cronbach's alpha reliability coefficient (for the categories of definitely preventable, potentially preventable, and non-preventable) showed good internal consistency (0.83 for overall preventable death and 0.86 for hospital preventable death). Cronbach's alpha reliability coefficient (for the two categories-preventable versus non-preventable) showed very high internal consistency (0.91 for overall preventable death and 0.90 for hospital preventable death).

\section{DISCUSSION}

This study demonstrates that a substantial portion of traumatic deaths in patients with traumatic brain injuries are either definitely or potentially preventable. Furthermore, almost all of these preventable deaths occurred at the hospital stage of care and such data can be used to improve future care of patients with head injuries.

Our determined rates of preventable death are slightly lower than previously reported by Kim et al. ${ }^{4}$ In that study, $29 \%$ of deaths were determined to be preventable. In the present study, preventable deaths were only considered to occur if there was a unanimous decision among reviewers. Thus, classifying preventable death into three categories may have produced slightly lower results. Establishing multi-investigator agreement in the likelihood of the possibility of preventable death and reclassifying preventability into two categories rather than three resulted in more internal consistency as expected.

We identified a small but measurable rate (4.9\%) of preventable death in the pre-hospital processes. The result in this study 
(4.9\%) was less than the $13.9 \%$ identified in Kim's study. ${ }^{4}$ Although primary traumatic brain injury occurs at the time of head injury, secondary brain injury can occur at a variety of times including the prehospital phase. Preventing secondary or delaying brain injury can substantially reduce the mortality rate. ${ }^{11}$ Errors identified in the prehospital transport included inadequate airway management, inadequate monitoring, inadequate cervical spine protection, and transport to an inappropriate hospital.

Initial guidelines for the treatment of brain injured patients were introduced in 1995 and continue to be revised based on current evidence. ${ }^{8,12}$ This includes guidelines for both the prehospital and hospital treatment of head injured patients. Following these guidelines improves survival rates. ${ }^{13}$ These treatment guidelines are not uniformly adopted in emergency medical facilities in South Korea; therefore, it is difficult to determine if the facilities are in accordance with guidelines for the treatment of patients with traumatic brain injury. In this study, the majority of errors occurred in the emergency department with the transfer phase being the second most common location. Thus, in order to reduce death rates due to traumatic brain injury, introduction, uptake, and application of treatment guidelines in the emergency department is necessary.

The study by Kim et al. ${ }^{4}$ suggested that as ICISS survival probability increased, preventable mortality increased. In this study, however, preventable mortality was not higher in those patients with a predicted survival $>75 \%$. In many countries, attempts are made to evaluate mortality by using Trauma and Injury Severity Score (TRISS) methodology. Expected ICISS probability is calculated from the ICD-10 empirical probability of survival, and is a substitute for the existing TRISS. ${ }^{14}$ Proposed categories using TRISS probability of survival include the following: less than $25 \%$ probability of survival in the non-preventable cohort, $25 \%$ to $50 \%$ in the potentially preventable cohort; and 50\% or more in the definitely preventable cohort. ${ }^{15,16}$ These studies, however, demonstrate a higher probability of observed survival. As medical treatment has advanced since the TRISS methodology was developed in the 1980s, applying TRISS to current data, likely results in the higher than expected survival rates. Current evidence does not support this classification of ICISS probability of survival, but a categorization system is needed to compare rates of preventable death.

We identified a substantial number of medical errors in the treatment of head injured patients (Table 3). Most errors occurred in the emergency department treatment, and many of these could have been prevented. Actual errors in the emergency department are related to the consultation and work-up process (Appendix 1). These errors may perhaps be related to emergency department system or overcrowding. The second most common location for errors was in the prehospital phase although these contributed to pa- tient mortality less often. We did not identify errors from the operating suite, although this likely reflects a limitation of the medical record review as opposed to an actual absence of errors. In this study, an important and measurable rate of preventable mortality was identified in head injured patients. Errors were common and most occurred in the emergency department. Furthermore, errors were common in the prehospital phase but did not always lead to mortality. When analyzed by type of problem, both process-related and structure-related factors occurred in similar proportions.

This study has a number of limitations. It is a retrospective medical record review subject to the limitations of that methodology. The sample size included was insufficient to statistically identify small differences. Second, the selected panelists' area of expertise is in emergency medicine, and the results may be impacted by this expertise. A neurosurgeon or trauma surgeon may identify additional cases including errors that occurred in the operating suite or intensive care unit, or may have evaluated the preventability of cases differently.

This study enrolled patients at five hospitals belonging to the Korean Traumatic Brain Injury Surveillance Network. Although including multiple centers increases generalizability, it does not necessarily represent the entire population. ${ }^{17}$ Despite these limitations, this study is the first study to calculate preventable mortality of severe traumatic brain injury patients. A larger multicenter study on patients with traumatic brain injury would further clarify the questions raised by this research.

In addition, it took several years to publish the results following our study. If possible, it would be valuable to incorporate more recent data and evaluate results from the larger population over a longer period.

Our small population study found that an important and measurable rate of preventable mortality occurs in the initial care of traumatic brain injury patients. Patient care errors were common, and most occurred in the emergency department. Errors were also common in the prehospital phase but did not always lead to mortality. When analyzed by type of problem, both process- and infrastructure-related errors occurred in similar proportions.

\section{CONFLICT OF INTEREST}

No potential conflict of interest relevant to this article was reported.

\section{ACKNOWLEDGMENTS}

Korea Centers for Disease Control and Prevention supported this study financially. 


\section{REFERENCES}

1. Statistics Korea. Statistics of the cause of death [Internet]. Daejeon: Statistics Korea; 2011 [cited 2015 Feb 15]. Available from: http://kosis.kr.

2. MacKenzie EJ. Review of evidence regarding trauma system effectiveness resulting from panel studies. J Trauma 1999;47 (3 Suppl):S34-41.

3. Jung KY, Kim JS, Kim Y. Problems in trauma care and preventable deaths. J Korean Soc Emerg Med 2001;12:45-56.

4. Kim Y, Jung KY, Cho KH, et al. Preventable trauma deaths rates and management errors in emergency medical system in Korea. J Korean Soc Emerg Med 2006;17:385-94.

5. Marion DM. Traumatic brain injury. New York: Thieme Medical Publishers; 1999.

6. Servadei F, Nanni A, Nasi MT, et al. Evolving brain lesions in the first 12 hours after head injury: analysis of 37 comatose patients. Neurosurgery 1995;37:899-906.

7. American College of Surgeons. Advanced trauma life support for doctors. 8th ed. Chicago, IL: American College of Surgeons; 2008.

8. Brain Trauma Foundation; American Association of Neurological Surgeons; Congress of Neurological Surgeons. Guidelines for the management of severe traumatic brain injury. J Neurotrauma 2007;24 Suppl 1:S1-106.

9. MacKenzie EJ, Steinwachs DM, Bone LR, Floccare DJ, Ramzy Al. Inter-rater reliability of preventable death judgments. The
Preventable Death Study Group. J Trauma 1992;33:292-302.

10. Association for the Advancement of Automotive Medicine. Abbreviated injury scale: 2005 revision. Barrington, Rl: Association for the Advancement of Automotive Medicine; 2008.

11. Lu J, Marmarou A, Choi S, Maas A, Murray G, Steyerberg EW. Mortality from traumatic brain injury. Acta Neurochir Suppl 2005;95:281-5.

12. Badjatia N, Carney N, Crocco TJ, et al. Guidelines for prehospital management of traumatic brain injury 2nd edition. Prehosp Emerg Care 2008;12 Suppl 1:S1-52.

13. Fakhry SM, Trask AL, Waller MA, Watts DD; IRTC Neurotrauma Task Force. Management of brain-injured patients by an evidence-based medicine protocol improves outcomes and decreases hospital charges. J Trauma 2004;56:492-9.

14. Kim Y, Jung KY, Kim CY, Kim YI, Shin YS. Validation of the International Classification of Diseases 10th edition based injury severity score (ICISS). J Prev Med Public Health 1999;32: 538-545.

15. Chiara O, Cimbanassi S, Pitidis A, Vesconi S. Preventable trauma deaths: from panel review to population based-studies. World J Emerg Surg 2006;1:12.

16. Mann NC, Mullins RJ, MacKenzie EJ, Jurkovich GJ, Mock CN. Systematic review of published evidence regarding trauma system effectiveness. J Trauma 1999;47(3 Suppl):S25-33.

17. Mullins RJ, Mann NC. Population-based research assessing the effectiveness of trauma systems. J Trauma 1999;47(3 Suppl): S59-66. 
Appendix 1. A list of actual errors by location and type

\begin{tabular}{|c|c|c|c|}
\hline No. of errors & Location of errors & Type of errors & Actual problems \\
\hline 2 & Prehospital & Process & Inadequate airway management \\
\hline 1 & Prehospital & Process & Inadequate cervical spine protection \\
\hline 4 & Prehospital & Structure & Transport of the patient to a hospital without operating suite available at that time \\
\hline 2 & Prehospital & Structure & Transport of the patient to a hospital without the department of neurosurgery \\
\hline 2 & Prehospital & Structure & Transport of the patient to a hospital without general surgeon available at that time \\
\hline 2 & Interhospital transfer & Process & Delay in transfer to another hospital \\
\hline 1 & Interhospital transfer & Structure & Inadequately staffed ambulance \\
\hline 1 & Interhospital transfer & Structure & Inadequately equipped ambulance \\
\hline 9 & Emergency department & Process & Refusal of operation consent by family member \\
\hline 4 & Emergency department & Process & Delay of blood transfusion \\
\hline 3 & Emergency department & Process & Inadequate airway management \\
\hline 5 & Emergency department & Structure & Delay of computed tomography scanning because of overcrowding in emergency department \\
\hline 4 & Emergency department & Structure & Delay in transfer to operating suite \\
\hline 2 & Emergency department & Structure & Delay of intensive care unit admission because of no available bed \\
\hline 2 & Emergency department & Structure & Lack of intracranial pressure monitoring resulting in abrupt brain herniation \\
\hline 1 & Emergency department & Structure & Absesce of neurosurgeon capable of craniotomy at that time \\
\hline 1 & Intensive care unit & Process & Delay in detecting ST segment elevation myocardial infarction on electrocardiogram \\
\hline 1 & Intensive care unit & Process & No deep vein thrombosis prophylaxis \\
\hline 1 & Intensive care unit & Structure & No device for renal replacement therapy available at that time \\
\hline
\end{tabular}


Appendix 2. Problem codes to investigate preventable death ${ }^{3,4}$
A. Prehospital
B. Emergency department
C. Operation room
D. Intensive care unit
E. General ward
F. Interhospital transfer
1. System inadequacy
1-1. Reception, delivery, consultation Inappropriate reception Inappropriate transport
1-2. Resuscitation
1-3. Investigation Delay in investigation
1-4. Monitoring Inadequate monitoring facilities Lack of intracranial pressure monitoring
1-5. Transfer
Delay in transfer to operation room
1-6. Others
2. Problems in treatment
2-1. Consultation
2-1-1 Failure or delay of neurosurgery consultation
2-1-2 Failure or delay of general surgery consultation
2-1-3 Failure or delay of chest surgery consultation
2-1-4 Failure or delay of any consultation
2-1-5 Inadequate observation
2-1-9 0ther inappropriate reception/delivery
2-2. Resuscitation
2-2-1 Inadequate airway resuscitation
2-2-2 Inadequate ventilation or breathing
2-2-3 Inadequate fluid resuscitation
2-2-4 Inadequate blood resuscitation
2-2-9 0ther resuscitation problems
2-3. Investigation or assessment error
Failure to arrange computed tomography of head
Delay in investigation
Failure to perform proper investigation
Inadequate assessment of abdomen
2-3-1 Head
2-3-2 Origin of shock
2-3-3 Chest or respiration
2-4. Monitoring
Inadequate arterial blood gas analysis or oxygen monitoring
Intracranial pressure monitoring
2-5. Transfer
Transfer delay to operation room
No laparotomy
Inappropriate transfer to ward premature
Discharge
Delay in transfer to operation room
Delay in transfer to another hospital
2-6. Other treatment
Inadequate cervical spine protection
Chest decompression delayed
Delay in fracture stabilization/fixation
No deep vein thrombosis prophylaxis
Inadequate nutrition
3. Problems in diagnosis
Epidural hemorrhage and subdural hemorrhage
Ruptured aorta
Perforated esophagus
Liver and spleen laceration
Delayed management of deep vein thrombosis
Failure of diagnosis of acute respiratory distress syndrome
Mesenteric ischemia
Head injury with chronic alcoholics
4. Problems in procedures
Excessive rapid medical anti-shock trousers deflation
Worsening hypovolemic shock
Inadequate IV lines
Inadequate respiratory resuscitation
Inappropriate anesthetic technique
Inappropriate operative procedures
Central venous pressure complication
Injured mesentery by suprapubic catheter
5. Others

2-3-4 Abdomen 